\title{
The Spatial Agglomeration and Industrial Network of Strategic Emerging Industries and Their Impact on Urban Growth in Mainland China
}

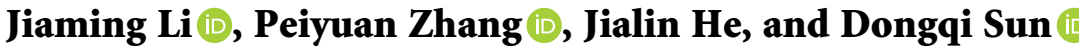 \\ Institute of Geographic Sciences and Natural Resources Research (CAS), 11A, Datun Road, Chaoyang District, \\ Beijing 100101, China \\ Correspondence should be addressed to Dongqi Sun; sundq@igsnrr.ac.cn
}

Received 3 May 2021; Accepted 8 August 2021; Published 31 August 2021

Academic Editor: Jing-Hu Pan

Copyright (c) 2021 Jiaming Li et al. This is an open access article distributed under the Creative Commons Attribution License, which permits unrestricted use, distribution, and reproduction in any medium, provided the original work is properly cited.

Strategic emerging industries (SEIs) are an important industrial policy to promote innovation, develop advanced manufacturing, and upgrade the economy in China. The research explores the impact of SEIs on urban economic growth in mainland China, from agglomeration externalities and network externalities. The results show that SEIs have a significant impact on growth, and network externalities are generally more important than agglomeration externalities. Although the agglomeration of large-scale listed enterprises in the city promotes growth, too many large enterprises in the cluster, that is, the lack of small and medium-sized supporting enterprises, will harm urban growth. Meanwhile, a city with a higher degree of centrality has a larger GDP, but the strength has a negative impact.

\section{Introduction}

Strategic emerging industries (SEIs) are those key industries that the Chinese government considers to have an important impact on global economic competition. Those, specifically, include 9 industries: new generation of information technology industry, high-end equipment manufacturing industry, new material industry, bioindustry, new energy automobile industry, new energy industry, energy conservation and environmental protection industry, digital creative industry, and related service industry. Since they were proposed in 2010, SEIs have received great attention from the government. "The Fourteenth Five-Year Plan for National Economic and Social Development of the People's Republic of China and the Outline of Long-Term Goals for 2035," a key document for China's development in the next five years, regards strategic emerging industries as an important part of China's modern industrial system and high-quality economic development.

In addition to the impact on the future development of China, more importantly, the dual characteristics of these industries are worthy of attention. On the one hand, 7 out of
9 industries belong to manufacturing, so the agglomeration economy, that promotes the rapid growth of manufacturing, has an impact on the outlay and development of SEIs. On the other hand, the importance of R\&D and information to the development of the industry makes these industries show a certain degree of characteristics of the network. The industrial network is usually a characteristic of the high-end service industry. For SEIs, are agglomeration economies and network externalities equally important? Or which one is more important? Actually, many advanced manufacturing industries are important; they are also affected by both the agglomeration economy and network externalities. This research has reference significance for thinking about the development of advanced manufacturing industries.

1.1. Literature Review. Agglomeration economy and network externalities are from two different theoretical frameworks. The former focuses on the mutual influence between enterprises within the region, while the latter pays more attention to the influence of the outside on the region. 
The benefits of local development from agglomeration economies mainly come from three aspects: close upstream and downstream industrial connections, knowledge and information spillover, and large-scale labor market pool [1-3]. To a large extent, Marshall believed that agglomeration economies were formed by specialized division of labor by different enterprises in the same industry. But in reality, the proximity of plants across industries also often occurs. Namely, those plants from different industries also benefit from being close to each other. Jacobs [4] argued the spatial agglomeration of diverse industries and people helps the emergence of new ideas, thereby promoting the development of the local economy. Ellison et al. [5] also developed coagglomeration indices and found some highly coagglomerated industry pairs in the United States. Studies have shown that coagglomeration of multiple industries is conducive to the development of emerging industries and hightech industries, while for traditional industries, it is mostly the agglomeration of enterprises in the same industry $[6,7]$. Industry clusters, formed by the agglomeration of related industries, are important for regional growth [8]. Not only are the industry clusters collections of enterprises and even public sectors, but they also emphasize the cooperation network formed between enterprises as an important source of competitive advantage.

Compared with the cooperation network based on spatial proximity in industrial clusters, network externality emphasizes the benefits from the information or financial connections outside the region or city. Therefore, network externality highlights the impact of nonlocalization. Rosenthal and Strange [9] also argued the effect of economic activities outside a certain area on the economic activities within that area should not be ignored. Certainly, the study of industrial agglomeration is not completely ignored by external influences. For example, the concept of market potential is introduced to measure the influence of other cities on one city. However, the calculation of market potential is still based on the distance between cities. So it is still not beyond spatial proximity and distance decay.

With the rise of globalization and flow space, interconnected agglomerations and cities have become the engine of economic growth [10,11]. Based on the Sassen [12] and Castells [13] elaboration of the global cities system, Taylor and Derudder [14] developed the interlocking network model (INM) to measure network externality. Urban network externalities are mostly from two aspects: function complementarities between cities and borrowed size. The former elaborates that functional networks between cities could create synergies and complementarities $[15,16]$. The latter showed the benefits that small and medium-sized cities get from large cities. When Alonso [17] first introduced the concept of borrowed size, it only explained that the agglomeration benefits that small and medium-sized cities acquired due to their proximity to large cities. Meijers and Burger [18] recently argued that the concept can be easily generalized to network connectivity between large and small cities. It means small and medium cities can gain benefits by being well-positioned in both geographic space and urban networks.
The researches of two strands of agglomeration externalities and network externalities are separated and there are few comparatively empirical studies. So it is unknown about the relative importance of agglomeration externalities visà-vis network externalities. The same is true for SEIs and other industries in China. Some studies have shown that strategic emerging industries are mainly concentrated in the economically developed eastern part of China, and their distribution centers are shifting southward [19, 20]. Zhao [21] showed the producer network in China. One of the purposes of the research is to answer which externalities are more important for ESIs and urban growth.

\section{Data and Methodology}

2.1. Data. The dataset of this study includes 1069 listed enterprises from Shanghai Stock Exchange (SHSE) and Shenzhen Stock Exchange (SZSE) in strategic emerging industries and their associated 18713 enterprises. China Securities Index Co., Ltd., and Shanghai Stock Exchange released 1117 sample enterprises included in Strategic Emerging Composite Index in 2017. We collected 1069 in the 1117 sample enterprises. Furthermore, we obtained the branches of these listed enterprises and the affiliated enterprises they invest in, by cooperating with Shanghai HeHe Information Technology Co., Ltd. Listed enterprises generally have strong strengths and can be regarded as core enterprises, leading the development of strategic emerging industries in China, while the branches and the affiliated enterprises can be taken as supporting ones. There are 1,900 sample enterprises in the research. Besides, we also obtained data such as GDP, employees, and fixed asset investment from the statistical yearbook.

2.2. Methodology. In general, this study did not use complex analysis methods. The analysis of industrial agglomeration is mainly based on the number of urban enterprises, while for network externality, three basic indicators, degree centrality, strength, and average degree of the neighbors, are measured [22].

\subsubsection{Degree Centrality}

$$
K_{i}=\sum_{j} a_{i j}, \quad j \in n, j \neq i,
$$

where the sum runs over the set $n$ of neighbors of $i$. If there are links between city $i$ and city $j$, then $a_{i j}=1$; otherwise, $a_{i j}=0$.

2.2.2. Strength. The strength of a node integrates the information about both its connectivity and the importance of the weights of its links.

$$
S_{i}=\sum_{j} a_{i j} * \omega_{i j}, \quad j \in n, j \neq i,
$$

where $a_{i j}$ is the same as above and $\omega_{i j}$ is the weight, which is the number of enterprise connections between city $i$ and city $j$. 
2.2.3. The Average Degree of the Neighbors. To identify the degree correlation of industry network, we need to measure the average degree of the neighbors of each city. The average degree of the neighbors is identified:

$$
\begin{aligned}
& N_{i}=\frac{1}{k_{i}} \sum_{j} a_{i j} * k_{j}, \quad j \in n, j \neq i, \\
& N_{i}^{\omega}=\frac{1}{k_{i}} \sum_{j} a_{i j} * \omega_{i j} * k_{j}, \quad j \in n, j \neq i .
\end{aligned}
$$

If high-degree cities tend to link with those cities with high-degree centrality, this tendency is referred to as an assortative network. Otherwise, tendency of high-degree/ low-degree cities and that of low-degree/high-degree neighbors to connect are referred to as disassortative ones [23].

\section{The Spatial Agglomeration Characteristics and Cluster Construction of China's SEIs}

Nearly 20,000 core and supporting enterprises are located in 329 prefecture-level and above cities and 24 counties directly administrated by the province in China. Among China's 333 prefecture-level administrative units, only 9 cities in a few provinces such as Tibet, Qinghai, and Xinjiang have no supporting enterprises. Specifically, 1069 core enterprises are distributed in 141 prefecture-level and above cities. Except for the 4 municipalities in China, more than $40 \%$ of the cities have core enterprises in SEIs; 18,843 supporting enterprises are distributed in 352 cities. Only 19 of the 293 prefecture-level cities have no supporting enterprises. Overall, SEIs are widely distributed in Chinese cities.

\subsection{Agglomeration and Distribution of Core Enterprises in} SEIs. Macroscopically, most cities in the eastern coastal region have core enterprises of SEIs. In the central and northeastern regions, they are mainly distributed in central cities and surrounding areas, while in the northwest, they are scattered in some cities such as Lanzhou and Urumqi. The spatial distribution of SEIs' core enterprises in the eastern, central, and western regions is uneven.

From the perspective of regional distribution and agglomeration, core enterprises are mainly concentrated in the Yangtze River Delta, Beijing-Tianjin-Hebei, Pearl River Delta, and Chengdu-Chongqing regions (Figure 1). Among them, core enterprises have the highest degree of agglomeration in the Yangtze River Delta. The number of core enterprises in the Yangtze River Delta has reached 308, accounting for $28.81 \%$ of the 1,069 core enterprises. In this region, there are 8 cities with more than 10 core enterprises. The number of core enterprises in the Beijing-Tianjin-Hebei region and the Pearl River Delta region is about 200 (209 and 193, respectively). However, the characteristics of spatial distribution are significantly different. In the Beijing-Tianjin-Hebei region, the distribution of core enterprises is very concentrated. More than $86 \%$ of core enterprises (180) are located in Beijing, while the number of core enterprises in
Tianjin is 15 , and in other cities, there are only $1-2$. The distribution of core enterprises in the Pearl River Delta is relatively even. There are more than 5 core enterprises in 7 cities, and 4 of them have more than 10 core enterprises. There are 48 core enterprises in the Chengdu-Chongqing region, mainly located in Chengdu and Chongqing (30 and 11, respectively).

Although core enterprises in SEIs are widely distributed in China's 142 cities, core enterprises are highly concentrated in a few cities and their municipal districts. More than $40 \%$ of the 142 cities have only one core enterprise (59 cities, accounting for $41.55 \%)$. And more than $50 \%$ of the core enterprises are concentrated in 8 cities including Beijing and Shenzhen (Table 1). Even in these big cities, the majority of core enterprises are concentrated in a few municipal districts. The agglomeration degree of core enterprises in SEIs at the county (district) level is significantly higher than that at the city level. Among the 2,846 county-level administrative units, only 387 have core enterprises, accounting for only $13.57 \%$. And there is only one in 223 counties (districts and county-level cities). The top five districts with the total number of core enterprises are Haidian District in Beijing, Nanshan District in Shenzhen, Bao'an District in Shenzhen, Chaoyang District in Beijing, and Pudong New District in Shanghai (the core enterprises are $85,42,34,33$, and 22, respectively). These top five municipal districts have gathered close to one-fifth of the country's core enterprises in SEIs (19.30\%).

\subsection{Agglomeration and Distribution of Supporting Enterprises} in SEIs. Beijing-Tianjin-Hebei region, Yangtze River Delta region, Pearl River Delta region, and Chengdu-Chongqing region are still the regions with the largest number of supporting enterprises (Figure 2). In the Yangtze River Delta and the Pearl River Delta, the number of supporting enterprises in most cities is relatively large, while the distribution of core enterprises shows the characteristics of the core-periphery in the Beijing-Tianjin-Hebei and ChengduChongqing regions. In the latter two regions, most supporting enterprises are concentrated in regional central cities, while the number of small and medium-sized cities is significantly smaller. In most of the central and western provinces, supporting enterprises of SEIs are also mainly concentrated in provincial capitals.

The cities with more than 1,000 supporting enterprises are still Beijing, Shanghai, and Shenzhen. The total number of supporting enterprises in the three cities is close to 5,000 (4711), accounting for $1 / 4$ of all supporting enterprises. There are 37 cities with more than 100 supporting enterprises. The total number of supporting enterprises in these cities reached 13,067 , accounting for nearly $70 \%$. It is worth noting that only 11 of these 37 cities are located in the north of China. In terms of SEIs, there is a big gap between northern cities and southern cities in China.

The scope of enterprise agglomeration is further measured. The results show the agglomeration space of core enterprises and supporting enterprises is the same, and the distances between every two enterprises are gathered in 


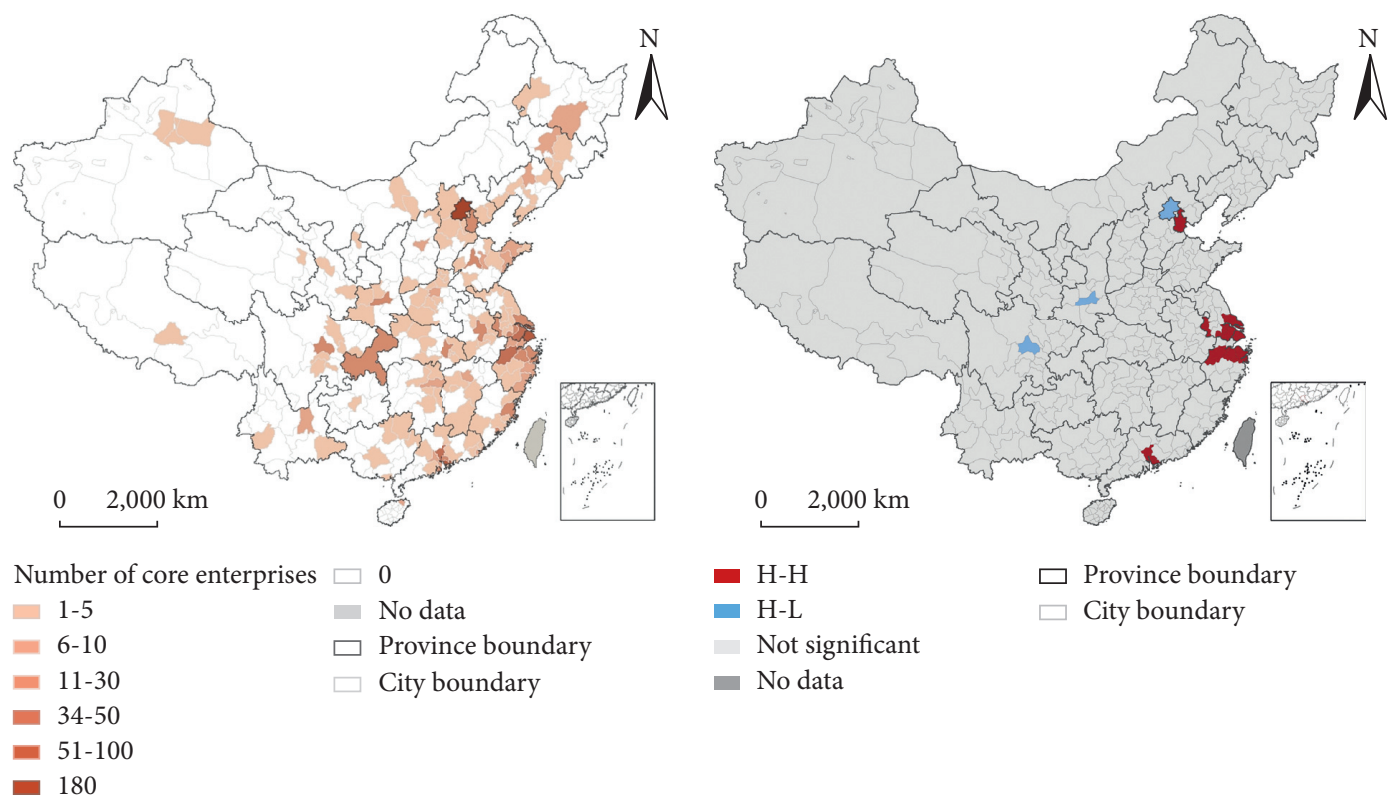

FIGURE 1: Spatial distribution and agglomeration of core enterprises in SEIs.

TABLE 1: Cities with 10 or more core enterprises in SEIs and their numbers.

\begin{tabular}{lccc}
\hline City & Number of core enterprises & City & Number of core enterprises \\
\hline Beijing & 180 & Ningbo & 15 \\
Shenzhen & 98 & Zhuhai & 15 \\
Shanghai & 91 & Jinan & 15 \\
Hangzhou & 47 & Xiamen & 14 \\
Suzhou & 39 & Hefei & 13 \\
Guangzhou & 34 & Fuzhou & 13 \\
Chengdu & 30 & Dongguan & 13 \\
Wuhan & 27 & Nantong & 12 \\
Nanjing & 21 & Shaoxing & 12 \\
Xi'an & 18 & Chongqing & 11 \\
Wuxi & 16 & Shenyang & 10 \\
Tianjin & 15 & & Top ten cities \\
\hline Percentage & Primate city & 56.22 \\
\hline
\end{tabular}

$90 \mathrm{~km}, 1100 \mathrm{~km}$, and $1900 \mathrm{~km}$ (Figure 3). (1) $90 \mathrm{~km}$ is roughly equal to the distance from a large city to a small city in its market hinterland but is significantly less than the distance between cities of $800-1300 \mathrm{~km}$. It means SEIs are mainly centered on regional central cities and are clustered in the surrounding $100 \mathrm{~km}$. The SEI industrial cluster, composed of large core enterprises and small and medium supporting enterprises, is mainly concentrated in the regional central city and its surrounding area about $100 \mathrm{ki}-$ lometers. (2) The second peak of the distances between every two enterprises is $1100 \mathrm{~km}$, which is just within the range of the distance between cities $(800 \mathrm{~km}-1300 \mathrm{~km})$. Furthermore, the distance between Beijing-Tianjin-Hebei or the Pearl River Delta and the Yangtze River Delta and ChengduChongqing area is about $1,000 \mathrm{~km}$. The industrial clusters are located in these urban agglomeration areas. So the $1100 \mathrm{~km}$ is the distance between industrial clusters of SEIs. (3) $1900 \mathrm{~km}$, the third peak, is roughly equivalent to the distance from the Beijing-Tianjin-Hebei to the Pearl River Delta. It also shows the distance between the distant industrial clusters in China. In general, large core enterprises and small and medium-sized supporting enterprises are concentrated on major urban agglomeration areas centered on large cities and develop several regional industrial clusters of SEIs in China.

3.3. The Structure and Distribution of Strategic Emerging Industrial Clusters. In addition to the number of enterprises, the structural characteristics presented by the proportions of different types of enterprises also have an important impact on the development of industrial clusters. Studies have shown there are Marshall-type industrial clusters dominated by small and medium-sized enterprises and wheel-type industrial clusters dominated by large, medium, and small enterprises. 


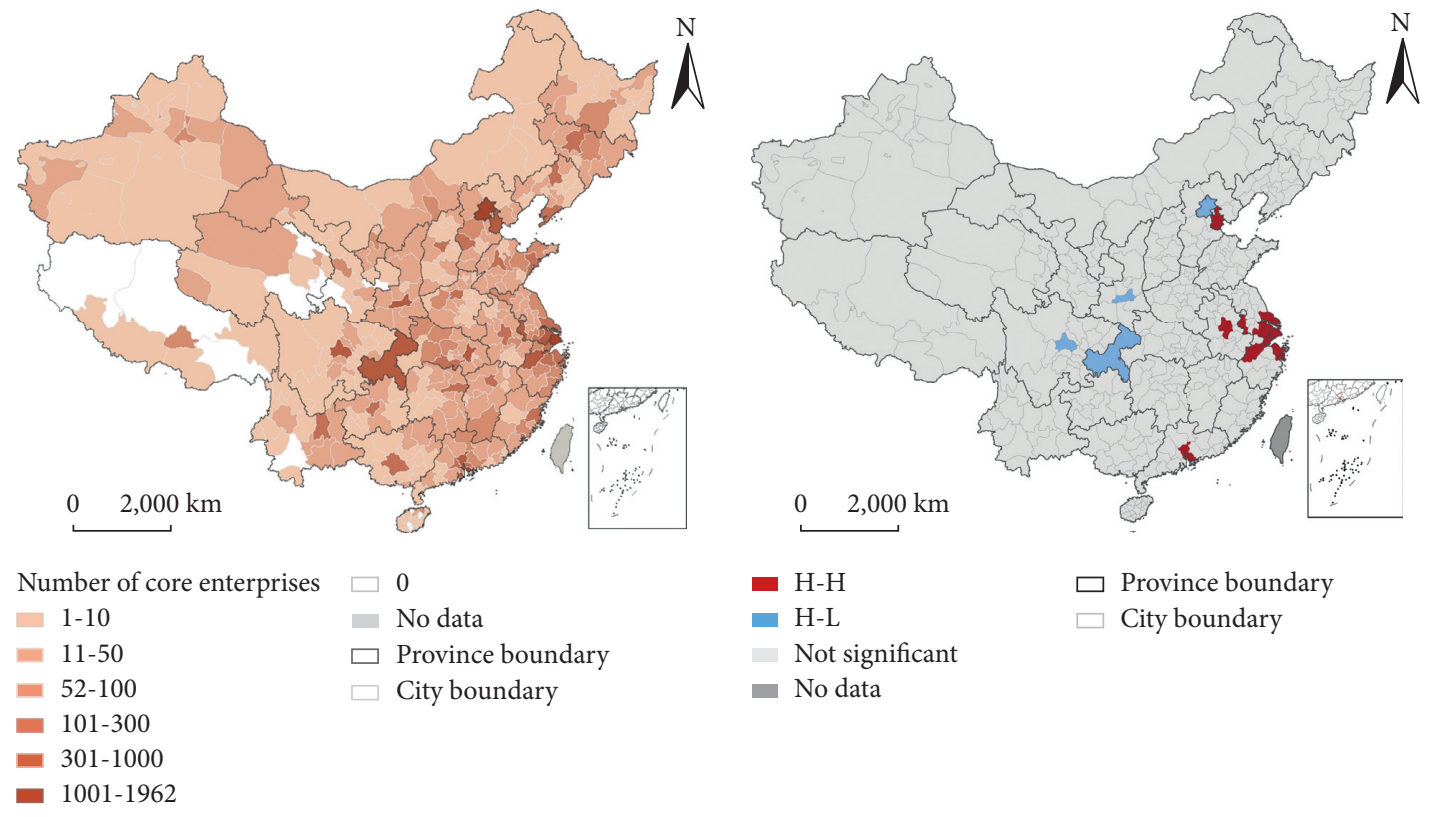

FIGURE 2: Spatial distribution and agglomeration of supporting enterprises in SEIs.

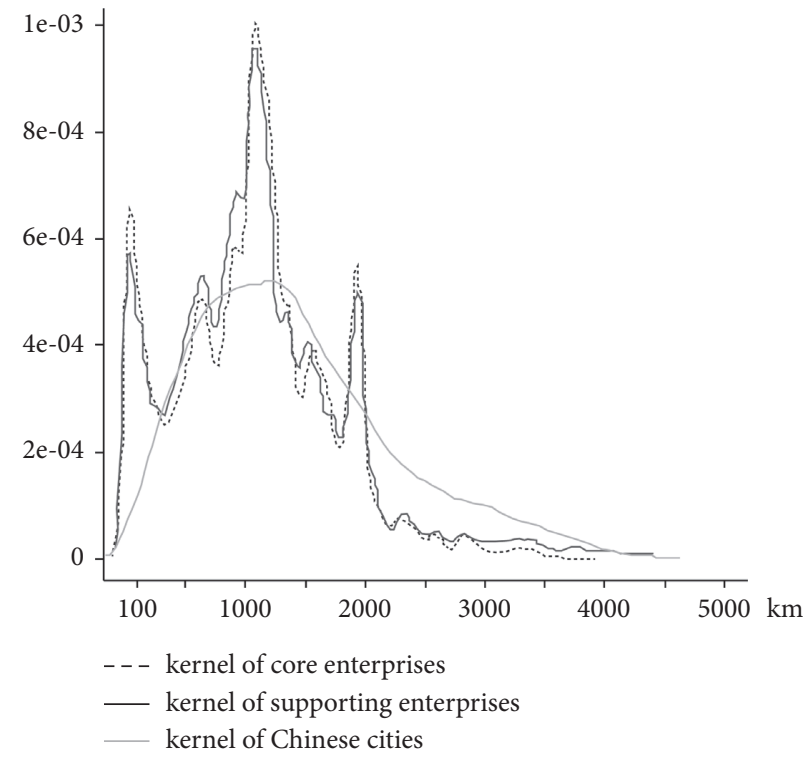

Figure 3: The spatial agglomeration characteristics of core enterprises and supporting enterprises.

The research will further analyze the cluster structure and characteristics of SEIs in different cities based on the proportions between large core enterprises and small and medium-sized supporting enterprises. The SME support ratio of clusters in Chinese cities (the ratio of the number of small and medium-sized supporting enterprises to the number of large core enterprises in the city) has a median number of 17 , and the lower quartile and upper quartile of the SME support ratio of each city are 12.17 and 27, respectively. According to this, cities can be divided into nonsupported type (cities with leading enterprises but no supporting enterprises), support-deficient type (cities with SME support ratio less than 12.17), balanced type (cities with
SME support ratio between 12.17 and 27), core-deficient type (cities with SME support ratio more than 27), and noncore type. Those cities with less than 5 enterprises related to SEIs are considered to be non-industrial cluster cities.

In terms of types, since core enterprises are mainly concentrated in central cities such as Beijing, Shanghai, and Shenzhen, nearly one-half of the cities are noncore or coredeficient types (Figure 4). There are 76 balanced cities, accounting for about $1 / 5$. Support-deficient and nonsupported cities account for less than $10 \%$. In addition, there are slightly more than one-fifth of cities that have not formed strategic emerging industrial clusters. It is worth noting that, contrary to what most people think, most large cities with 


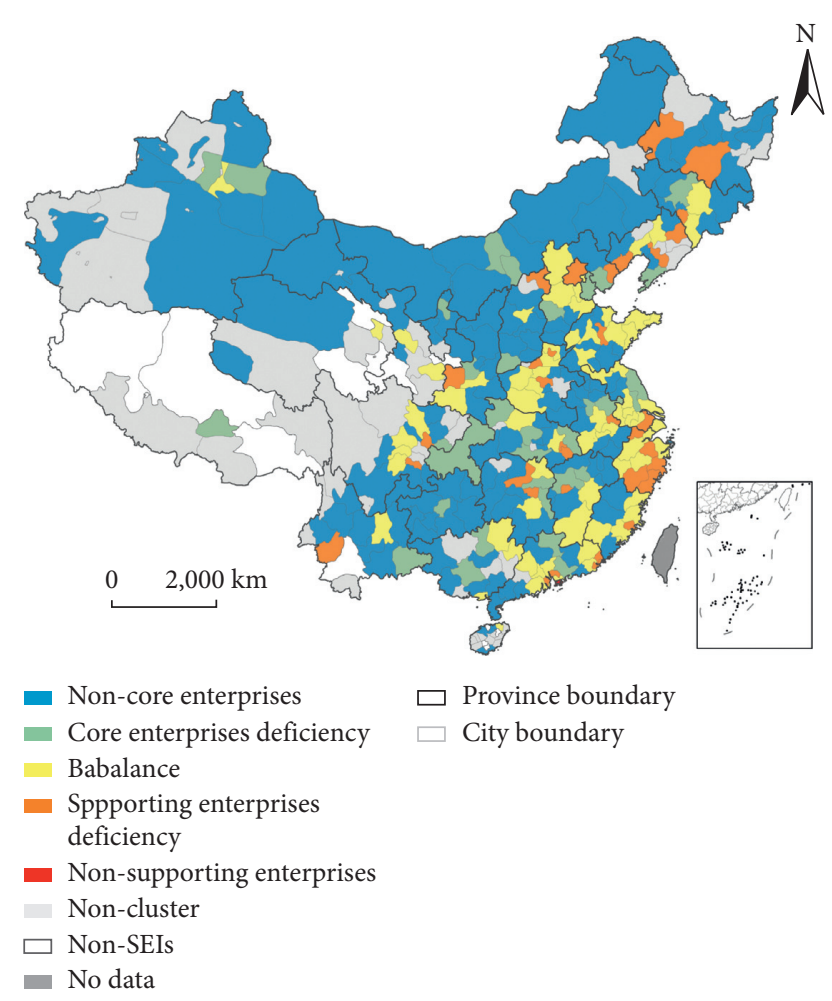

FIgURE 4: Spatial distribution of strategic emerging industrial clusters in China.

more core enterprises are balanced. There are not many cases of insufficient supporting enterprises due to the development of core enterprises. Actually, among the 23 cities with more than 10 core enterprises, only Beijing, Suzhou, Dongguan, and Shaoxing have a relatively lower SME support ratio. Tianjin and Chongqing even have a relatively lower proportion of core enterprises. The core enterprises and supporting enterprises in most cities are relatively balanced. Most the support-deficient cities are due to undeveloped industrial clusters. Half of the cities of this type have less than 20 supporting enterprises, and 8 cities have 10 or fewer supporting enterprises.

In terms of the distribution of various cities, the relatively developed urban agglomeration areas along the eastern coast such as the Beijing-Tianjin-Hebei, Yangtze River Delta, Pearl River Delta, Shandong Peninsula, and the west coast of the Straits have a relatively good ratio of core and supporting enterprises, mainly belonging to balanced and support-deficient types. Only in the fringe areas far from regional central cities, there are a small number of coredeficient and noncore cities and a certain degree of coreperiphery distribution.

In the near western (Chongqing, Sichuan, and Shaanxi) and central provinces, different types of cities are mixed. There are fewer balanced cities, and most cities are coredeficient and noncore. In particular, there are 14 core-deficient cities in the 8 provinces in these regions, accounting for more than $40 \%$ (43.75\%) of this type of city. From the perspective of the distribution of various types of cities, Anhui and Jiangxi, which are close to the coastal areas, have a trend of coordinated development with urban agglomeration of the Yangtze River Delta and the west straits coast. In general, some strategic emerging industrial clusters have been formed in the near western and central regions, but these clusters still suffered from insufficient core enterprises.

For far western and northeastern provinces, western Sichuan and Hainan are dominated by noncore and noncluster cities. Only 5 of the 64 noncluster cities are not in these regions. Most of the noncore cities also have undeveloped clusters. There are nearly 70 noncore cities in these regions. More than $60 \%$ of cities have less than 10 supporting enterprises, and only three cities have more than 20 supporting enterprises. Among the 9 support-deficient cities, only Harbin and Anshan have more than 20 related enterprises. In other cities, the number of enterprises is relatively small. In the fringe areas of western and northeastern China, the development of strategic emerging industries is relatively slow.

\section{Networking Characteristics of China's SEIs}

Although dominated by manufacturing, SEIs are cuttingedge, innovative, and rapid iterative. Therefore, factors such as information, $\mathrm{R} \& \mathrm{D}$, and capital are also crucial to the development of SEIs. Among the 18,843 supporting enterprises invested by 1069 core enterprises of SEIs, nearly 7,000 are related to information and $\mathrm{R} \& \mathrm{D}$, accounting for nearly $40 \%(37.10 \%)$, and the number of financial and business service enterprises is 2,738, accounting for nearly $15 \%(14.53 \%)$, while manufacturing enterprises account for only $20 \%(20.16 \%)$. Different from the manufacturing process, the allocation of elements such as information, $\mathrm{R} \& \mathrm{D}$, and capital is not only localized but has obvious network characteristics.

4.1. Network Structure of SEIs. From the perspective of the overall structure of the SEIs network, SEIs present a diamond pattern with Beijing, Shanghai, Guang-Shen (Guangzhou, Shenzhen), and Chengdu as the apex. In the diamond-type industrial connection network, the strength of connections between cities in the east of the BeijingGuang-Shen line is significantly higher than that in the west. This result is consistent with the conclusions of some urban network research [24].

Degree centrality and strength, two key indicators of network analysis, represent different meanings in the study of industrial linkages. Degree centrality reflects the extensiveness of a city's connections across the country. For strength, it is the sum of the number of industrial connections between a city and other cities (represented by the number of enterprises connected between cities in this study) and reflects the total frequency of connections with other cities. More importantly, this indicator of a city is significantly affected by a few key cities that have large-scale industrial connections with the city.

The results show that Beijing's degree centrality reaches 297, indicating that Beijing has industrial connections with another 332 prefecture-level administrative units in China, 
followed by Shenzhen's degree of 194, and Shanghai's degree of 163 (Figure 5). The top ten cities in terms of extensive connections all have more than 100 degrees. Similar to the result of degree centrality, Beijing, Shenzhen, and Shanghai are still the three cities with the highest strength $(3815,1717$, and 1580, respectively). But the divergence of the strength between cities is more obvious than that of degree centrality. For example, the values of the strength between Shenzhen and Shanghai is only $45 \%$ and $41 \%$ of Beijing's, respectively; and Hangzhou, the fourth-highest city, has a connection strength of only 922 , which is also less than $60 \%$ of the thirdhighest city, Shanghai.

Generally speaking, Beijing is the most advantageous city in the industrial connection network. The values of both the degree and strength are much larger than in other cities including Shenzhen and Shanghai. Besides, those cities with a higher degree of centrality generally have higher strength, but there are also slight differences. For example, Dongguan, which ranks seventh in degree, ranks 18th in strength, while Tianjin, which ranks 12th in degree, ranks among the top 10 in strength.

The industrial connection network formed by degree centrality and the strength differ particularly in terms of matching. In the topological network formed by the SEIs, the degree centrality and its corresponding average degree of the neighbors show an obvious negative correlation, and the correlation coefficient is $-0.848(P \leq 0.01)$ (Figure 6). It means cities with high degrees tend to be linked to cities with low degrees. So, from the perspective of degree centrality, the entire network is disassortative. It is exactly the opposite of the result of the network weighted by contact frequency. The global weight matching coefficient is 0.559 . So, from the perspective of strength, the weighted network is assortative (Figure 7). Cities connected with high-strength cities have a higher average strength than their neighbors. This result shows that when only considering the industrial links between cities, the SEIs network has a center-periphery structure. Small and medium-sized cities tend to have connections with large cities such as Beijing, Shenzhen, and Shanghai, while the connections between small and medium-sized cities are not close, showing the characteristics of vertical connections. When the number of connected enterprises is used as the weight, the large-scale and highstrength corporate connections between large cities have greatly increased the average strength of the neighbors, resulting in the weighted network showing the characteristics of assortativity. Therefore, the disassortativity of the topological network is mainly caused by the fact that small and medium-sized cities are less interconnected and mainly connected with large cities, while the assortativity of the weighted network is caused by the large-scale company connections between large cities. However, this does not change the overall structural characteristics of the hierarchical vertical connection industrial network. In general, the SEIs network presents multiple local networks with vertical connections centered on large cities, and the core cities in the local network are closely connected with high intensity.
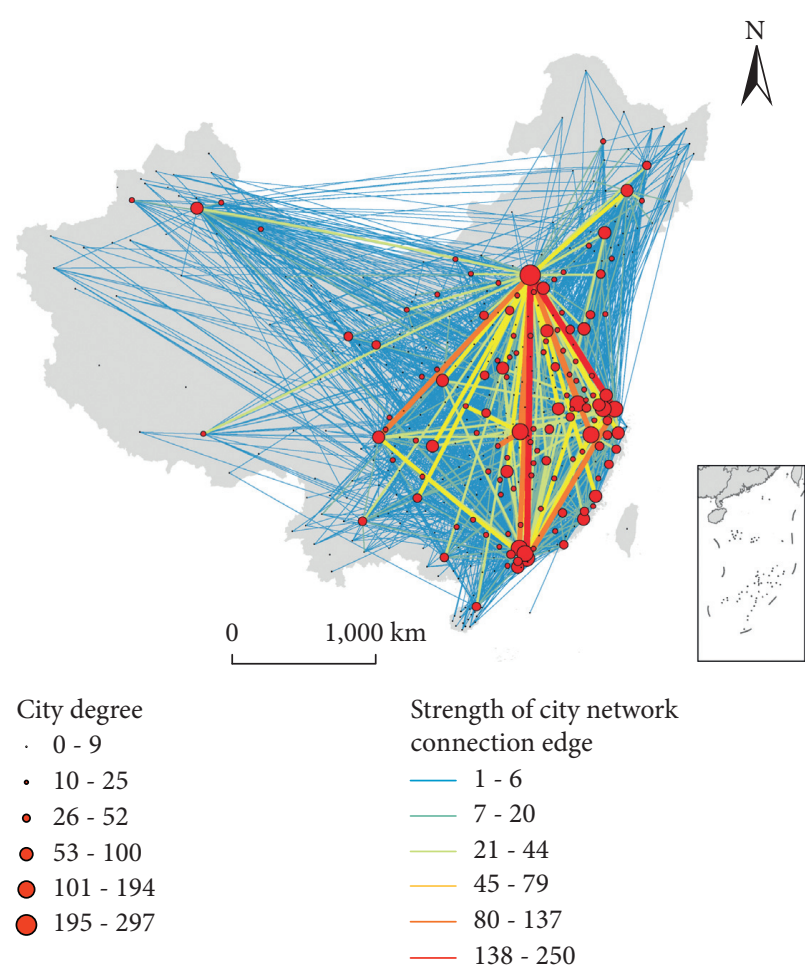

Figure 5: Network characteristics of China's SEIs.

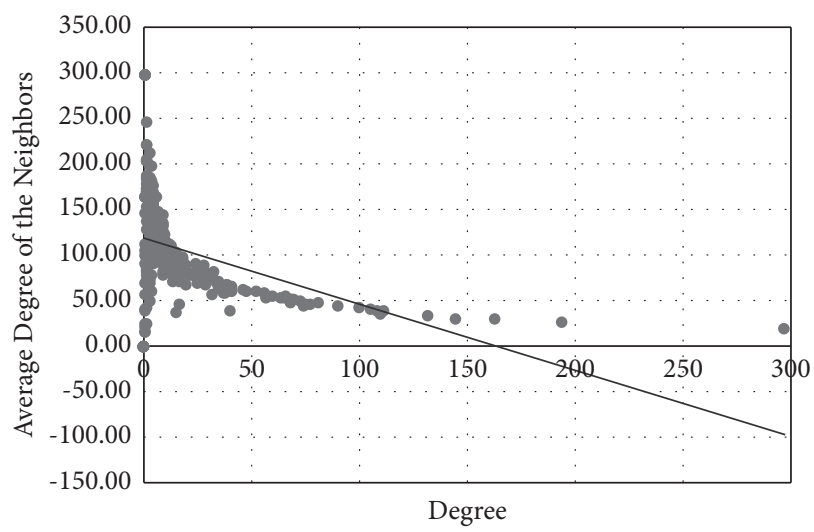

FIGURE 6: Degree correlation of the topological network of China's SEIs.

4.2. Industrial Connection Network of Major Node Cities. To further clarify the differentiated characteristics of different cities in the industrial connection network, a comparative analysis will be made on the industrial connection network of major cities. For Beijing, its investment in external regions is focused on central cities in the eastern and central regions (Figure 8). For example, Beijing has a high investment scale in Shanghai, Wuhan, Xi'an, Chengdu, and Shenzhen. However, Beijing has no obvious investment tendency in neighboring areas such as Hebei, Shandong, and Shanxi. Except for Tianjin that has invested in more than 100 related enterprises, cities such as Xi'an, Jinan, Shijiazhuang, and Shenyang and other cities have invested in less than 60 


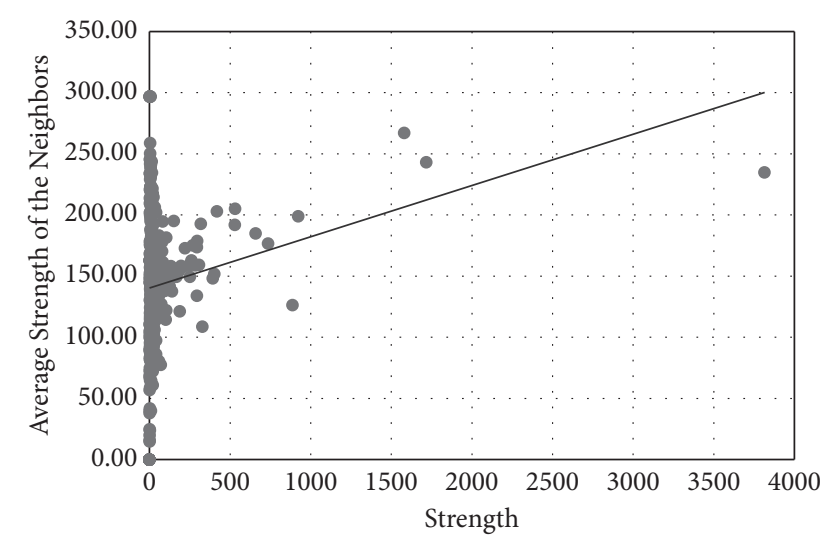

FIGURE 7: Strength correlation of the topological network of China's SEIs.

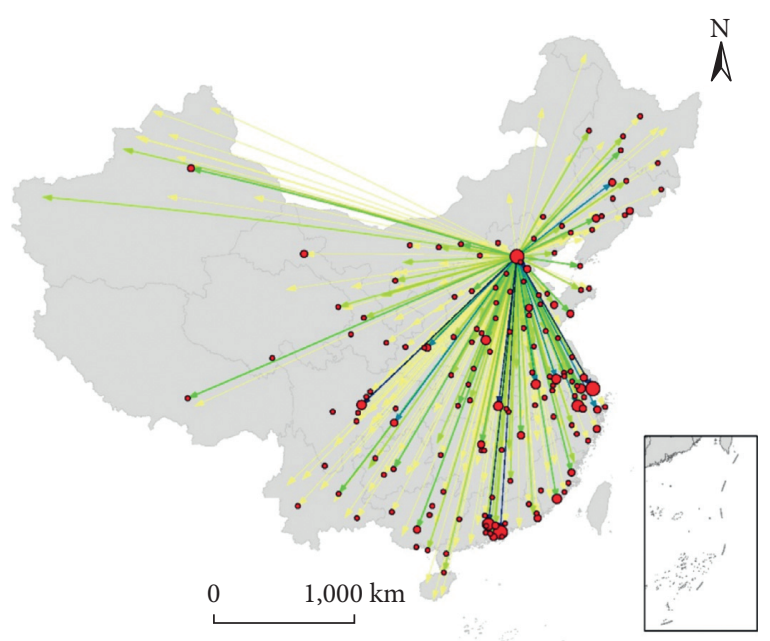

Beijing

Scale of investment in external regions (number of enterprises)

$\rightarrow 1-4$

$\longrightarrow 5-15$

$\longrightarrow 16-35$

$\longrightarrow 36-65$

$\longrightarrow 66-157$

Figure 8: The scale and direction of Beijing's SEIs investment in external regions.

related enterprises. The largest number of investment enterprises in the northern central city is Xi'an, which has invested in 58 enterprises, ranking 9th in the number of enterprises investing in external regions, and lower than central and southwestern central cities such as Wuhan and Chengdu.

Shenzhen is the second-largest node city after Beijing in China's SEI system. Except for its investment enterprises ranked second, Shenzhen also has a greater degree of investment across the country than Shanghai (Figure 9). Only $16.03 \%$ of enterprises investing outside Shenzhen are concentrated in the Pearl River Delta region, and among 22 cities with more than 10 invested enterprises from Shenzhen, only 4 cities are located in the Pearl River Delta area. At the same time, cities with a large number of enterprises invested

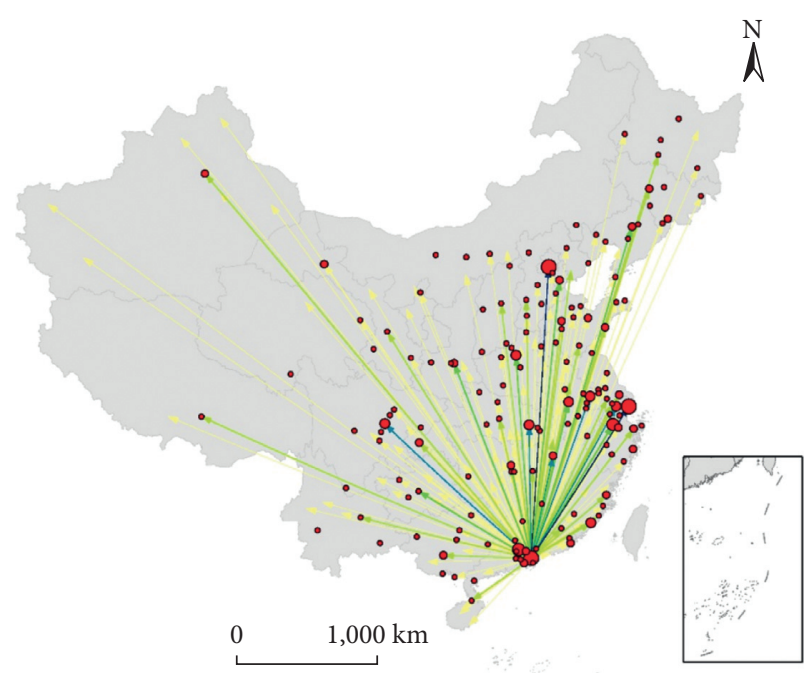

Shenzhen

Scale of investment in external regions (number of enterprises)

$1-4$

$\longrightarrow 5-15$

$\longrightarrow 16-35$

$\longrightarrow 36-65$

$\longrightarrow 66-117$

FIgURE 9: The scale and direction of Shenzhen's SEIs investment in external regions.

by Shenzhen include not only major urban agglomeration areas such as the Yangtze River Delta, the Pearl River Delta, Beijing-Tianjin-Hebei, and Chengdu-Chongqing, but also central cities in the northeast and southwest regions such as Shenyang and Kunming.

Apart from investing in large-scale enterprises in Beijing and Shenzhen, Shanghai's main investment cities are located in the Yangtze River Delta such as Jiangsu and Nanjing (Figure 10). There are 18 cities with more than 10 invested companies from Shanghai, and half is located in the Yangtze River Delta. Unlike Beijing's investment across all over the country, invested enterprises from Shanghai are mainly concentrated in the Yangtze River Delta region, accounting for $62.70 \%$. Nearly one-third of the enterprises investing outside Shanghai is located in the Yangtze River Delta region (32.95\%). There are fewer companies from Beijing investing in the Beijing-Tianjin-Hebei region. Less than 1/3 (32.75\%) of invested companies from Beijing are located in the Beijing-Tianjin-Hebei region, and even less than $10 \%$ $(8.14 \%)$ in Tianjin and Hebei province. In addition, Wuhan in the middle reaches of the Yangtze River and Chengdu and Chongqing in the upper reaches are also cities where Shanghai tended to invest.

Except for Beijing, Shenzhen, and Shanghai, other cities take their provinces as the main contact areas, so these cities are more inclined to be provincial-level central nodes. For example, four of the top seven cities that have the largest number of enterprises that Guangzhou invests in external regions, are the Pearl River Delta and surrounding areas (Foshan, Shenzhen, Changsha, and Shaoguan). Among the top 5 cities that have the largest number of enterprises that 

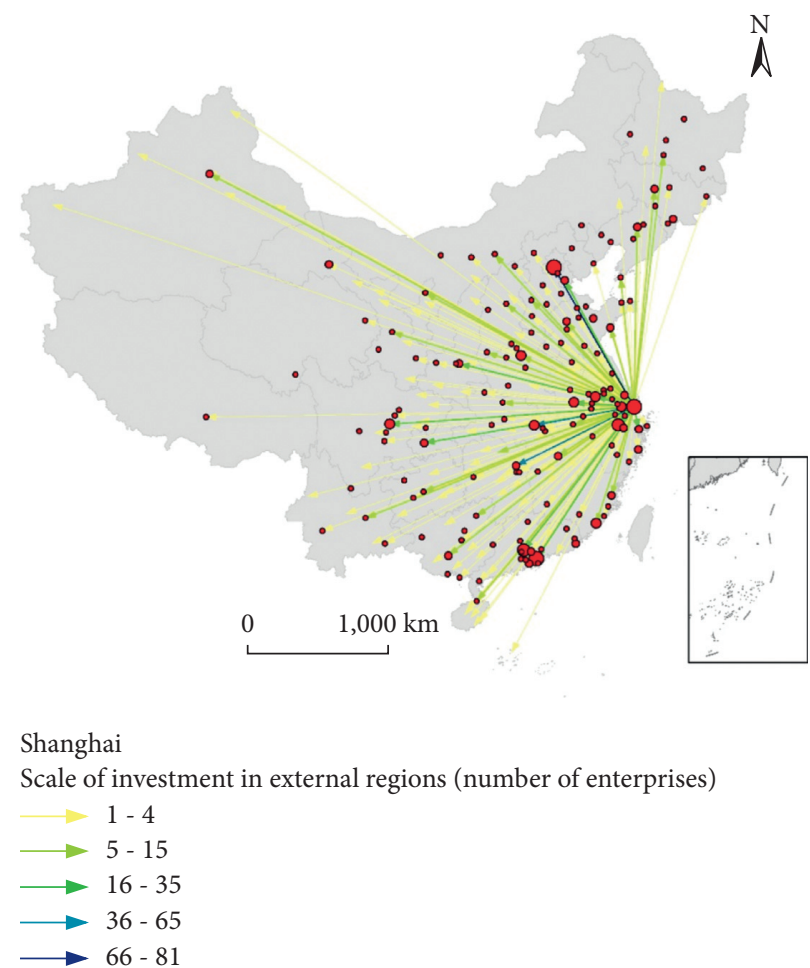

FIGURE 10: The scale and direction of Shanghai's SEIs investment in external regions.

Hangzhou invests in, only Beijing is not a city in Zhejiang Province. The number of enterprises investing in Hangzhou and other cities in Zhejiang Province accounts for $55.42 \%$ of the total of invested enterprises from Hangzhou. There are 13 cities with more than 10 enterprises invested in Nanjing (Figure 11). Among them, Beijing, which ranks second, and fifth Shenzhen, are not cities in Jiangsu Province.

Beijing is not only an export destination for investment by enterprises related to SEIs across the country but also an absorption destination for related investments. Beijing is the city with the largest number of enterprises invested by Shanghai, Shenzhen, and Nanjing, and the second-largest number of enterprises invested by Guangzhou and Nanjing. Therefore, Beijing plays a very important role in the construction of China's SEIs system and the process of industrial development, and it is also an important national node city in the industrial system. Shenzhen is second only to Beijing in the degree of industrial connections in China and is a quasi-national node city. Shanghai has the characteristics of a certain regional node city because its industrial connections are mainly concentrated in the Yangtze River Delta. The industrial connection network of other cities takes the province where it is located as the main spatial scope.

\section{The Impact of Spatial Agglomeration and Industrial Networks on Industrial Development}

To further clarify the influence of industrial agglomeration and industrial network on urban development, a multiple regression model is built based on the Cobb Douglas production function to analyze the influence of four variables, including cluster size, cluster structure, degree, and strength on urban economic growth. The model is as follows:

$$
\begin{aligned}
\ln Y= & \ln K+\ln L+\ln \text { Scale_large }+\ln \text { Structure } \\
& +\ln \text { Degree }+\ln \text { Strength },
\end{aligned}
$$

where $Y$ is GDP in 2018, $K$ is capital stock in 2017, $L$ is the number of employees in 2017, Scale_large is the number of core enterprises in SEIs, and Structure is the cluster structure of SEIs (specifically, the number of core enterprises/the number of supporting enterprises), Degree is the degree centrality of the city, and Strength is the strength of the city (Table 2).

The results show that the agglomeration and network of SEIs have a significant impact on urban economic growth. Among them, the number of core enterprises and degree of centrality have obvious positive effects on urban economic growth, while the cluster structure and the strength have negative effects (Table 3). From the perspective of industrial agglomeration and clusters, core enterprises have a significant positive impact, indicating that the more the core enterprises, the stronger the promotion of urban growth. However, the higher the proportion of core enterprises is, the less conducive they are to urban economic growth. The result shows the important role of supporting enterprises in SEIs clusters. At the same time, when the variable of the total number of enterprises in SEIs is added to the model, we find that the total number of enterprises has no significant effect on urban economic growth. In other words, the scale of SEIs clusters has little effect on urban growth. 

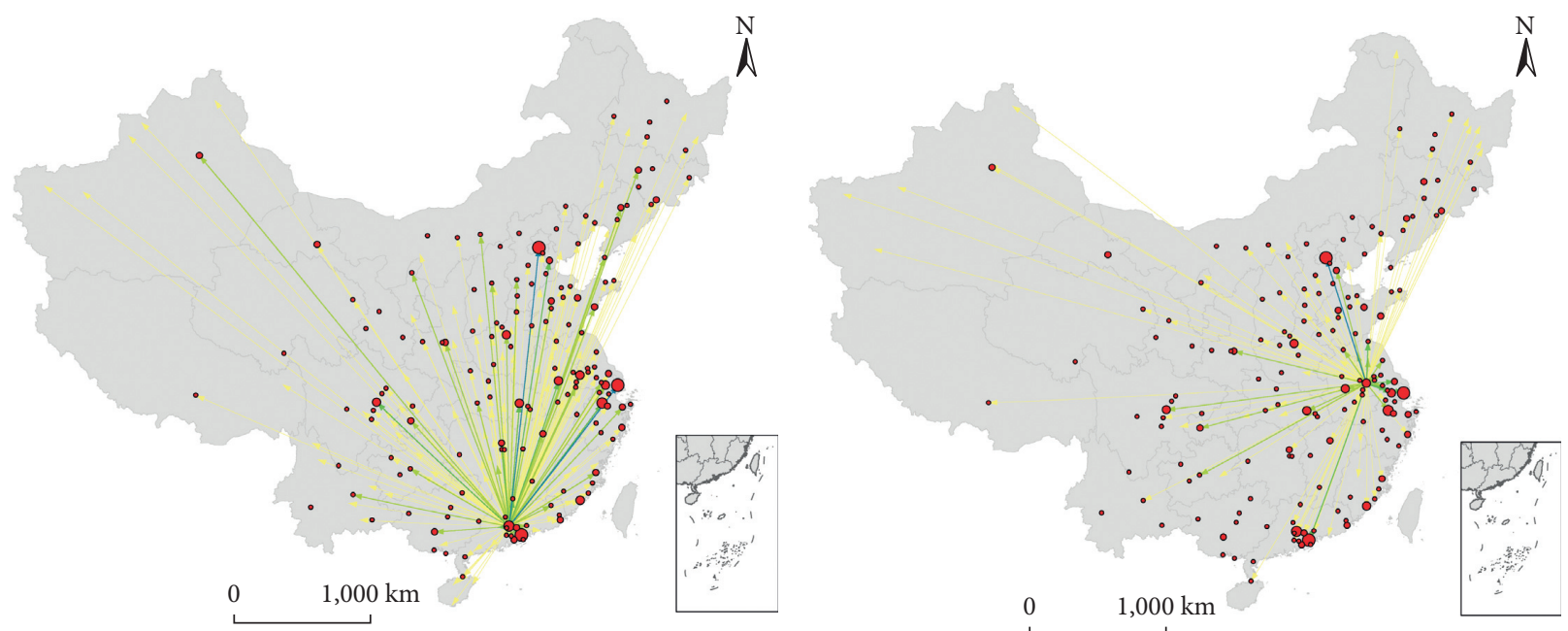

Guangzhou

Scale of investment in external regions (number of enterprises)

\section{Nanjing}

Scale of investment in external regions

(number of enterprises)
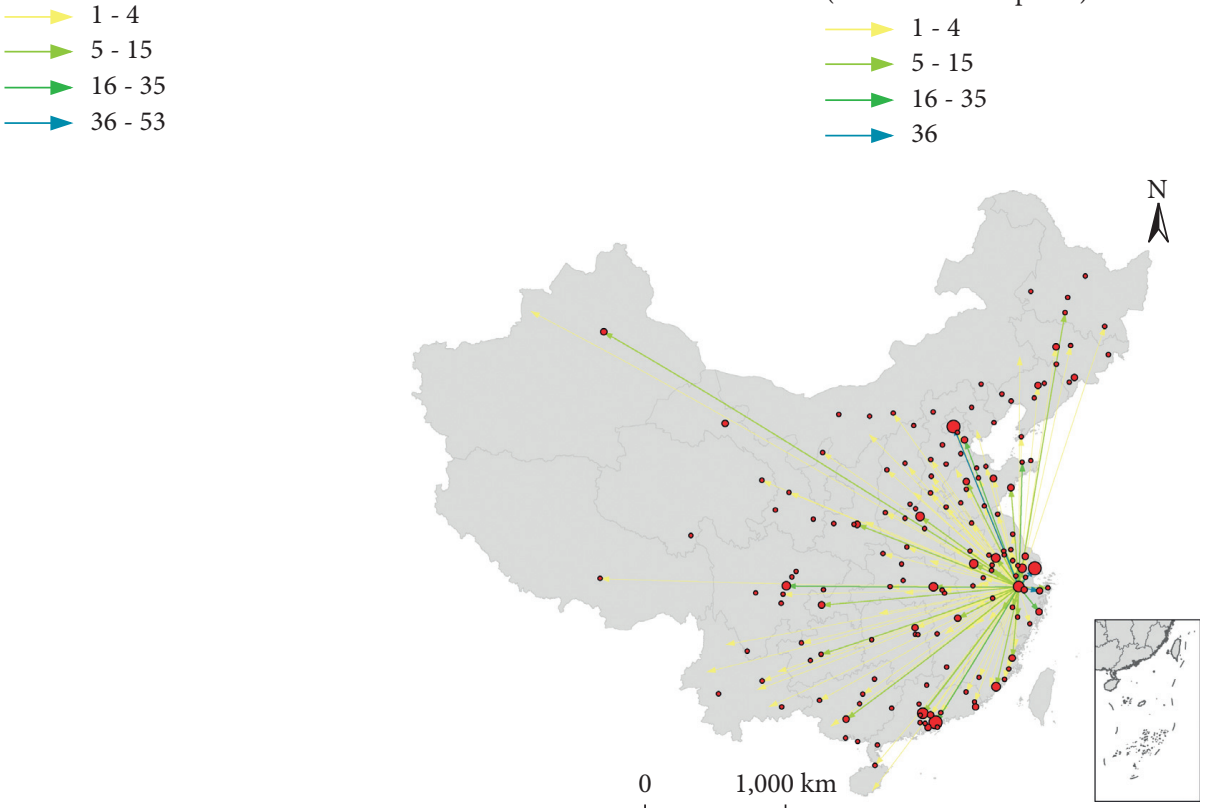

Hangzhou

Scale of investment in external regions

(number of enterprises)

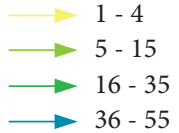

FIGURE 11: The scale and direction of SEIs investment of Guangzhou, Nanjing, and Hangzhou in external regions.

TABle 2: Descriptive statistics of cities.

\begin{tabular}{lccccccc}
\hline & $\begin{array}{c}\text { GDP in 2018 } \\
\text { (billions) }\end{array}$ & $\begin{array}{c}\text { Capital stock in } \\
\text { 2017 (billions) }\end{array}$ & $\begin{array}{c}\text { Number of employees } \\
\text { in 2017 (thousands) }\end{array}$ & $\begin{array}{c}\text { Number of large } \\
\text { enterprises in cities } \\
(n)\end{array}$ & $\begin{array}{c}\text { Cluster structure of } \\
\text { strategic emerging } \\
\text { industry }(/)\end{array}$ & $\begin{array}{c}\text { Degree } \\
(n)\end{array}$ & $\begin{array}{c}\text { Node } \\
\text { strength }(n)\end{array}$ \\
\hline Mean & 465.30 & 901.57 & 891.19 & 7.05 & 0.05 & 31.84 & 150.17 \\
Std. & 544.07 & 819.95 & 1142.99 & 19.23 & 0.04 & 40.02 & 387.16 \\
dev & 3267.99 & 5254.88 & 8128.59 & 180.00 & 0.23 & 297.00 & 3815.00 \\
Max & 27.42 & 66.70 & 83.19 & 0.00 & 0.00 & 1.00 \\
Min & & & & & & & 1.00 \\
\hline
\end{tabular}


TABLE 3: Regression results of industrial agglomeration, cluster structure, and linkage network.

\begin{tabular}{|c|c|c|c|c|}
\hline & Model 1 & Model 2 & Model 3 & Model 4 \\
\hline K & $\begin{array}{c}0.5770^{* * *} \\
(0.000)\end{array}$ & $\begin{array}{c}0.5092^{* * *} \\
(0.000)\end{array}$ & $\begin{array}{c}0.5523^{* * *} \\
(0.000)\end{array}$ & $\begin{array}{c}0.5138^{* * *} \\
(0.000)\end{array}$ \\
\hline$L$ & $\begin{array}{c}0.5316^{* * *} \\
(0.000)\end{array}$ & $\begin{array}{c}0.5268^{* * *} \\
(0.000)\end{array}$ & $\begin{array}{c}0.5269^{* * *} \\
(0.000)\end{array}$ & $\begin{array}{c}0.5240^{* * *} \\
(0.000)\end{array}$ \\
\hline Scale_large & & $\begin{array}{c}0.0741^{* *} \\
(0.047)\end{array}$ & & $\begin{array}{c}0.1211^{* *} \\
(0.013)\end{array}$ \\
\hline Structure & & $\begin{array}{c}-0.5208 \\
(0.327)\end{array}$ & & $\begin{array}{c}-1.0768^{*} \\
(0.056)\end{array}$ \\
\hline Degree & & & $\begin{array}{c}0.1369^{* *} \\
(0.023)\end{array}$ & $\begin{array}{c}0.1565^{* *} \\
(0.012)\end{array}$ \\
\hline Strength & & & $-0.0804^{*}(0.095)$ & $\begin{array}{c}-0.1470^{* * *} \\
(0.007)\end{array}$ \\
\hline$R^{2}$ & 0.9497 & 0.9505 & 0.9509 & 0.9525 \\
\hline
\end{tabular}

From the perspective of the industrial network, the degree centrality has a positive effect, and the influence of degree is the most stable among the four variables. It means that the extensiveness of the city's connections in the industrial network has a significant role in promoting urban economic growth. But the strength has a negative effect.

However, compared with capital stock and employees, the impact of strategic emerging industries on urban economic growth is still relatively small. The $R$ square of the two variables relative to GDP reaches 0.9497 , while the $R$ square only increases by 0.0028 after adding the four variables. To some extent, SEIs is still not a core driving force for urban economic growth.

\section{Concluding Remarks}

SEIs are a relatively special type of industry. On the one hand, the SEIs are dominated by manufacturing industries such as information equipment, new energy, and medicine, which are characterized by a relative agglomeration of manufacturing industries in nearby regions. On the other hand, because these industries require a large amount of capital, information, and R\&D investment, they also show the networked characteristics of high-end service industries. Based on the relevant data of China's SEIs, this paper deeply analyzes the characteristics of industrial cluster agglomeration and networking and finds that both the spatial agglomeration and networking of SEIs have a significant impact on the development of Chinese cities.

Enterprises related to SEIs are widely distributed in Chinese cities, but they are also highly concentrated. More than $40 \%$ of cities have core enterprises in SEIs, and more than $93 \%$ of prefecture-level cities have at least one supporting company. However, most core and supporting enterprises are concentrated in a few cities, such as Beijing, Shanghai, and Shenzhen, and a few regions such as the Yangtze River Delta, Beijing-Tianjin-Hebei, Pearl River Delta, and Chengdu-Chongqing region. The spatial distribution of both core enterprises and supporting enterprises presents a center-periphery model centered on large cities, and they are mainly concentrated within a range of $90 \mathrm{~km}$ around large cities. At the same time, China has formed several large industrial clusters, based on major urban agglomeration areas.

From the perspective of industrial clusters, the proportion between core enterprises and supporting enterprises in the eastern region is more balanced. The central region generally shows the characteristics of insufficient core enterprises, while the western and northeastern regions have no core or support company due to undeveloped industrial clusters. At the same time, noncluster cities with less than 5 related enterprises are also mainly distributed in the northeast, western ethnic minority areas, and Hainan.

From the perspective of the network, the diamond network pattern of SEIs is the same as that of the whole industry, but the development level of the western ChengduChongqing region is relatively low. Beijing, Shenzhen, and Shanghai are the first-tier cities in the industrial network. Among them, Beijing occupies a core position in China's strategic emerging industrial network. Nearly 300 cities have industrial connections with Beijing. Shenzhen also has the potential to become a national center. Relatively speaking, Shanghai is more inclined to be the center city of the Yangtze River Delta and the Yangtze River Basin, and more than 60\% of its external investment enterprises are located in the Yangtze River Delta region. Other cities, such as Guangzhou, Hangzhou, Chengdu, etc., are more regarded as provincial central cities, and their industrial connections are mostly within their provinces.

In general, both industrial agglomeration and the network of SEIs have a significant impact on the growth of cities. In particular, the extensiveness of the industrial connection network plays an important role in promoting the growth of cities, followed by the agglomeration of core enterprises in cities. However, it does not dwarf the importance of small and medium-sized supporting enterprises. An excessively high proportion of large core enterprises in industrial clusters harms urban economic growth.

\section{Data Availability}

The enterprises data used to support the findings of this study may be released upon application to the Shanghai $\mathrm{HeHe}$ Information Technology Co., Ltd., which can be contacted at the URL: https://www.qixin.com/?from=baidusemBrand1. 


\section{Conflicts of Interest}

The authors declare that they have no conflicts of interest.

\section{References}

[1] G. Ellison and E. L. Glaeser, "Geographic concentration in U.S. Manufacturing industries: a dartboard approach," Journal of Political Economy, vol. 105, no. 5, pp. 889-927, 1997.

[2] P. Krugman, "Increasing returns and economic geography," Journal of Political Economy, vol. 99, no. 3, pp. 483-499, 1991.

[3] A. Marshall, Principles of Economics, Macmillan, London, UK, 1920.

[4] J. Jacobs, The Death and Life of Great American Cities, Vintage Books, New York, NY, USA, 2012.

[5] G. Ellison, E. L. Glaeser, and W. R. Kerr, "What causes industry agglomeration? Evidence from coagglomeration patterns," The American Economic Review, vol. 100, no. 3, pp. 1195-1213, 2010.

[6] G. Duranton and D. Puga, "Nursery cities: urban diversity, process innovation, and the life cycle of products," American Economic Review, vol. 91, 2001.

[7] V. Henderson, A. Kuncoro, and M. Turner, "Industrial development in cities," Journal of Political Economy, vol. 103, 1995.

[8] E. J. Feser, Industry Clusters and Economic Development: A Learning Resource, University of Illinois, Illinois, IL, USA, 2004.

[9] S. S. Rosenthal and W. C. Strange, "Evidence on the nature and sources of agglomeration economies," Handbook of Regional and Urban Economics, vol. 4, pp. 2119-2171, 2004.

[10] R. Florida, Who's Your City? How the Creative Economy in Making where to Live the Most Important Decision of Your Life, Basic Books, New York, NY, USA, 2008.

[11] P. McCann and Z. J. Acs, "Globalization: countries, cities and multinationals," Regional Studies, vol. 45, pp. 17-32, 2011.

[12] S. Sassen, The Global City, Princeton University Press, Princeton, NJ, USA, 1991.

[13] M. Castells, The Rise of the Network Society, Blackwell, Oxford, UK, 1996.

[14] P. J. Taylor and B. Derudder, World-city Network: A Global Urban Analysis, Routledge, London, UK, 2nd edition, 2016.

[15] R. Boix and J. Trullén, "Knowledge, networks of cities and growth in regional urban systems," Papers in Regional Science, vol. 86, no. 4, pp. 551-574, 2007.

[16] R. Capello, "The city network paradigm: measuring urban network externalities," Urban Studies, vol. 37, no. 11, pp. 1925-1945, 2000.

[17] W. Alonso, "Urban zero population growth," Daedalus, vol. 104, 1973.

[18] E. J. Meijers and M. J. Burger, "Stretching the concept of "borrowed size"," Urban Studies, vol. 54, no. 1, pp. 269-291, 2017.

[19] J. Hu and Y. L. Zhao, "Research on agglomeration degree and its changing tendency of China's strategic emerging industries-empirical evidence from listed enterprises," Reform of Economic System, no. 6, pp. 102-106, 2015.

[20] C. A. Tang, L. J. Zhang, and Q. Yin, "Evolution and optimization of spatial layout of strategic emerging industry in China," Economic Geography, vol. 5, pp. 101-107, 2018.

[21] M. Zhao, X. Liu, B. Derudder, Y. Zhong, and W. Shen, "Mapping producer services networks in mainland Chinese cities," Urban Studies, vol. 52, no. 16, pp. 3018-3034, 2015.
[22] S. Boccaletti, V. Latora, Y. Moreno et al., "Complex networks: structure and dynamics," Complex Systems and Complexity Science, vol. 424, 2006.

[23] M. Newman, "The structure and function of complex networks," Proceedings of the National Academy of Sciences, vol. 101, no. 1, pp. 5200-5205, 2004.

[24] K. Wu, C. L. Fang, and M. X. Zhao, "The spatial organization and structure complexity of Chinese intercity networks," Geographical Research, no. 4, pp. 711-728, 2015. 